Article

\title{
Enhancing Energy Efficiency of a 4-DOF Parallel Robot Through Task-Related Analysis ${ }^{\dagger}$
}

\author{
Lorenzo Scalera ${ }^{1, *(1)}$, Paolo Boscariol ${ }^{2}$, Giovanni Carabin ${ }^{3}\left[\right.$, , Renato Vidoni $^{3}(\mathbb{C}$ and \\ Alessandro Gasparetto ${ }^{1}$ \\ 1 Polytechnic Department of Engineering and Architecture, University of Udine, 33100 Udine, Italy; \\ alessandro.gasparetto@uniud.it \\ 2 Department of Management and Engineering, University of Padova, 36100 Vicenza, Italy; \\ paolo.boscariol@unipd.it \\ 3 Faculty of Science and Technology, Free University of Bozen-Bolzano, 39100 Bolzano, Italy; \\ giovanni.carabin@unibz.it (G.C.); renato.vidoni@unibz.it (R.V.) \\ * Correspondence: lorenzo.scalera@uniud.it \\ + This paper is an extended version of our paper published in Vidussi, F., Boscariol, P., Scalera, L., Gasparetto, \\ A., Energetic analysis of industrial robots for pick-and-place operations. In Proceedings of the 25th \\ Jc-IFToMM Symposium, 2nd International Jc-IFToMM Symposium, Kanagawa, Japan, 26 October 2019.
}

Received: 6 February 2020; Accepted: 21 February 2020; Published: 25 February 2020

\begin{abstract}
Enhancing energy efficiency is one of the main challenges of today's industrial robotics and manufacturing technology. In this paper a task-related analysis of the energetic performance of a 4-DOF industrial parallel robot is presented, and the optimal location of a predefined task with respect to the robot workspace is investigated. An optimal position of the task relative to the robot can indeed reduce the actuators' effort and the energy consumption required to complete the considered operation. The dynamic and electro-mechanical models of the manipulators are developed and implemented to estimate the energy consumption of a parametrized motion with trapezoidal speed profile, i.e., a pick-and-place operation. Numerical results provide energy consumption maps that can be adopted to place the starting and ending points of the task in the more energy-efficient location within the robot workspace.
\end{abstract}

Keywords: robotics; dynamic model; parallel robot; task-related analysis; energy efficiency

\section{Introduction}

The development of state-of-the-art robotics and industrial manufacturing systems requires the adoption of high energy-efficient machines, especially when dealing with high speed operations performed for long periods of time. The reduction of energy consumption is a fundamental and critical aspect from both the economical and the ecological points of view. This is testified by the policies set by the European Union, which aims at supporting the adoption of energy-efficient improvements in order to reduce the energy consumption up to $30 \%$ by 2030 [1,2]. In this framework, the manufacturing industry is responsible for a large amount of the overall worldwide energy consumption [3], and this trend is foreseen to increase, given that the global annual sales of industrial robots is expected to grow by $+12 \%$ per year on average from 2020 to 2022 [4]. For these reasons, enhancing energy efficiency is becoming a challenging problem for industrial engineers and academic researchers.

The importance of this topic is testified by a flourishing literature, which includes both theoretical and experimental investigations on energy saving in automatic machines and robots [5]. Among the several strategies that can be followed to achieve the goal of enhancing the energetic performance of a manufacturing system, the main are: the proper choice of the robot architecture [6], the design of robots 
and machine with lightweight links and components [7-10], the substitution of traditional drives with regenerative ones [11-13], and the exploitation of the system natural dynamics [14-16]. Energy saving can also be achieved by optimizing the motion time and properly planning the trajectories that the machine will follow [17-19]. Examples of trajectory planning strategies, with the aim of reducing the torque or energy expenditure of robot actuators, comprise the analytical analysis of standard primitives for point-to-point motions [20,21], the exploitation of kinematic redundancy [22,23], and the adoption of algorithms for motion design with constrained paths [24-26].

Energy efficiency can also be enhanced by evaluating the performance of the robot throughout its workspace and by optimizing the location of the task with respect to the base of the manipulator. The positioning of the robot with respect to the required task is indeed essential not only for the correct execution of the operation, such as for example in robotic painting [27-29] or pick-and-place operations $[15,30,31]$ but also to minimize the expenditure of energy required by the actuators.

In this context, several local and global performance metrics have been proposed in the literature to investigate the behavior of industrial machines and robots. Local performance measures can indeed be adopted to define possible relations between robot design feature and the task to be executed. Moreover, local performance metrics can guide the choice of the position of the task relative to the robot, but also provide an analysis of the performance of the manipulator with respect to its workspace [32,33]. Examples of performance metrics for robot manipulators are given by [34,35], where different indexes are applied to a SCARA robot and graphically visualized. Furthermore, in [36], a task-dependent performance index is proposed, which explicitly accounts for both robot kinematics and task geometrical features.

In this paper a task-related analysis of the energetic performance of a four-leg delta-like (4-RUU) parallel manipulator is presented. The lightweight structure allows the robot to be employed for high-speed operations, especially for pick-and-place purposes [36,37]. The dynamic and electro-mechanical models of the manipulator are presented and adopted to estimate the actuators energy expenditure needed to perform a predefined basic operation within the robot workspace. Numerical results provide energy consumption maps that can be adopted as guidelines for the optimal robot positioning.

The work is the extended version of a preliminary conference paper presented in [38]. It is organized as follows: Section 2 reports the kinematic and dynamic models of the robot, Section 3 introduces the electro-mechanical model of the actuators, and in Section 4 the task-dependent analysis is described. Results are reported in Section 5, whereas Section 6 gives the conclusions of the work.

\section{Model of the 4-DOF Parallel Robot}

In this section the dynamic model of the 4-DOF parallel robot considered in this paper is recalled. The robot, shown in Figures 1 and 2, allows three translations and one rotation of the end-effector around its vertical axis. The end-effector is attached to a mobile platform, which is split into two parts connected by two linkages, defining a four-bar linkage that allows a rotation of the end-effector within $\pm \pi / 4 \mathrm{rad}$, as can be seen in Figure 3 . The range of rotation of the fourth degree of freedom $\vartheta$ is then amplified by means of a gearbox up to $\pm \pi \mathrm{rad}$.

The geometrical and dynamic parameters of the parallel robot used in this work are reported in Tables 1 and 2, respectively. In particular, the parameters of the actuators considered in the electro-mechanical model are collected in Table 2. 


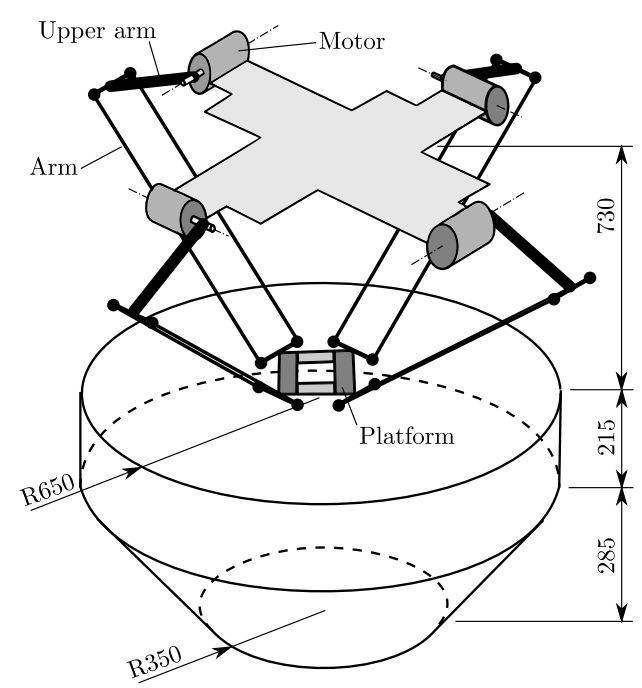

Figure 1. The 4-DOF parallel robot and its workspace limits in $\mathrm{mm}$.

\subsection{Kinematics}

The inverse kinematic relationship presented here allows to evaluate the joint variables $\boldsymbol{q}=\left[q_{1} q_{2} q_{3} q_{4}\right]^{T}$ from the end-effector pose $\boldsymbol{X}=\left[\begin{array}{ll}x y z & \vartheta\end{array}\right]^{T}$. This relationship can be derived by considering the closure equation of the mechanism:

$$
r_{l, i}^{T} r_{l, i}=r_{l, i}^{2}
$$

where

$$
\boldsymbol{r}_{l, i}=\boldsymbol{B}_{\boldsymbol{i}}-\boldsymbol{A}_{\boldsymbol{i}}=\left[\begin{array}{lll}
r_{l, i x} & r_{l, i y} & r_{l, i z}
\end{array}\right]^{T}
$$

The coordinates of points $\boldsymbol{A}_{\boldsymbol{i}}$, as used in Equation (2), are evaluated starting from the fixed points $\boldsymbol{P}_{i}$, known from the robot geometry as functions of the joint variables $\boldsymbol{q}_{i}$ and constant $r_{u, i}$, as shown in Figure 2. On the other hand, the coordinates of $\boldsymbol{B}_{\boldsymbol{i}}$ are obtained from the end-effector pose $\boldsymbol{X}$, by considering the constants $l, l_{1}, h$ and $h_{1}$ (Figure 3). Starting from Equation (2), the following system of equations can be obtained:

$$
I_{i} \sin \left(q_{i}\right)+L_{i} \cos \left(q_{i}\right)+K_{i}=0, \quad i=1, \ldots, 4
$$

The constants $I_{i}, L_{i}$ and $K_{i}$ depend on the geometry of the robot and on the end-effector pose [37]. They can be calculated as follows:

$$
\begin{aligned}
& I_{i}=2\left(z+z_{e}-z_{P i}\right) r_{u, i} \\
& L_{i}=-\sqrt{2}\left(M_{i} \operatorname{sign}\left(x_{P i}\right)+N_{i} \operatorname{sign}\left(y_{P i}\right)\right) r_{u, i} \\
& K_{i}=M_{i}^{2}+N_{i}^{2}+\left(z+z_{e}-z_{P i}\right)^{2}+r_{u, i}^{2}-r_{l, i}^{2}
\end{aligned}
$$

where $M_{i}$ and $N_{i}$ are defined as:

$$
\begin{aligned}
& M_{i}=x-\varepsilon_{i} l-\operatorname{sign}\left(y_{P i}\right) \frac{h}{2} \sin \frac{\vartheta}{4}+\operatorname{sign}\left(x_{P i}\right) \Delta l_{i}-x_{P i} \\
& N_{i}=y+\operatorname{sign}\left(y_{P i}\right) \frac{h}{2} \cos \frac{\vartheta}{4}+\operatorname{sign}\left(y_{P i}\right) \Delta h_{i}-y_{P i}
\end{aligned}
$$

$\left[x_{P i} y_{P i} z_{P i}\right]^{T}$ represents the coordinates vector of point $P_{i}$, whereas $z_{e}$ is the offset along the z-axis between the end-effector plane and the plane that contains points $B_{i}$ and $F_{i}$ (see Figure 3).

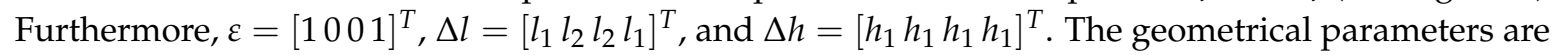
shown in Figure 3 and their numerical values are reported in Table 1. 


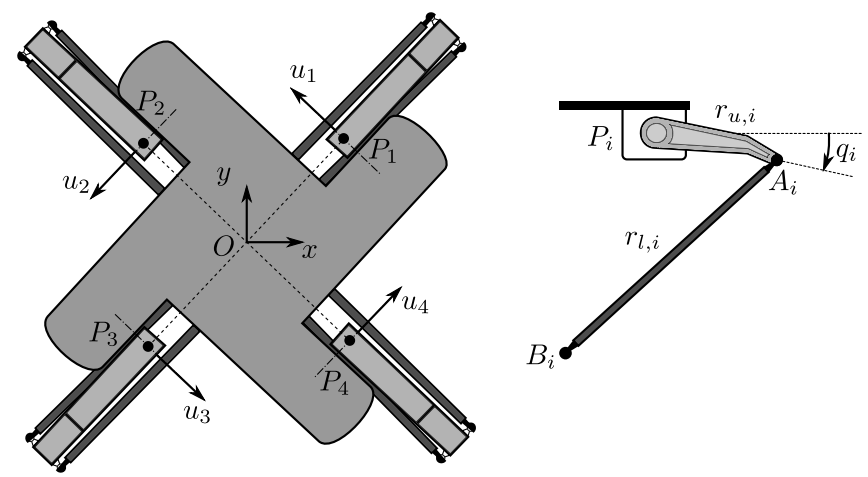

Figure 2. Notation used in the model of the parallel robot.

The solution of the inverse kinematics for the $i$-th joint variable $q_{i}$ assumes the following form:

$$
q_{i}=2 \arcsin \left(\frac{-I_{i} \pm \sqrt{\Delta_{i}}}{K_{i}-L_{i}}\right), \quad i=1, \ldots, 4
$$

where $\Delta_{i}=I_{i}^{2}-K_{i}^{2}+L_{i}^{2}$.

By considering the forward kinematics of the 4-DOF parallel manipulator, which aims at calculating the pose vector $X$ by knowing the joint variables vector $\boldsymbol{q}$, the problem leads to an eight-order polynomial in the fourth variable $\vartheta$. In this case, a numerical iterative scheme can solve the problem more efficiently than by searching the solutions in an analytical way [37].

The inverse velocity kinematics defines the relationship between the velocity vector of the end-effector $\dot{X}$ and the joint velocity vector $\dot{\boldsymbol{q}}$ :

$$
\dot{q}=J_{q}{ }^{-1} J_{X} \dot{X}=J^{-1} \dot{X}
$$

where $J$ indicates the Jacobian matrix of the manipulator, and matrices $J_{q}$ and $J_{X}$ are defined as:

$$
\begin{gathered}
\boldsymbol{J}_{\boldsymbol{q}}=\left[\begin{array}{cccc}
\boldsymbol{u}_{\mathbf{1}}{ }^{T}\left(\boldsymbol{r}_{\boldsymbol{u}, \mathbf{1}} \times \boldsymbol{r}_{\boldsymbol{l}, \mathbf{1}}\right) & 0 & 0 & 0 \\
0 & \boldsymbol{u}_{\mathbf{2}}{ }^{T}\left(\boldsymbol{r}_{\boldsymbol{u}, 2} \times \boldsymbol{r}_{\boldsymbol{l}, 2}\right) & 0 & 0 \\
0 & 0 & \boldsymbol{u}_{3}{ }^{T}\left(\boldsymbol{r}_{\boldsymbol{u}, 3} \times \boldsymbol{r}_{l, 3}\right) & 0 \\
0 & 0 & 0 & \boldsymbol{u}_{4}{ }^{T}\left(\boldsymbol{r}_{\boldsymbol{u}, \mathbf{4}} \times \boldsymbol{r}_{l, 4}\right)
\end{array}\right] \\
\boldsymbol{J}_{\boldsymbol{X}}=\left[\begin{array}{cccc}
x_{b 1} & y_{b 1} & z_{b 1} & -\frac{1}{8} \boldsymbol{e}_{z}{ }^{T}\left(\boldsymbol{h} \times \boldsymbol{r}_{\boldsymbol{l}, \mathbf{1}}\right) \\
x_{b 2} & y_{b 2} & z_{b 2} & -\frac{1}{8} \boldsymbol{e}_{z}{ }^{T}\left(\boldsymbol{h} \times \boldsymbol{r}_{l, 2}\right) \\
x_{b 3} & y_{b 3} & z_{b 3} & \frac{1}{8} \boldsymbol{e}_{z}{ }^{T}\left(\boldsymbol{h} \times \boldsymbol{r}_{l, 3}\right) \\
x_{b 4} & y_{b 4} & z_{b 4} & \frac{1}{8} \boldsymbol{e}_{\boldsymbol{z}}{ }^{T}\left(\boldsymbol{h} \times \boldsymbol{r}_{l, 4}\right)
\end{array}\right]
\end{gathered}
$$

$u_{i}$ is the unit vector parallel to motor axis, $h=F_{3}-F_{2}$, and $e_{z}$ is the vertical axis of the absolute reference frame.

Finally, the inverse acceleration analysis computes the relationship between the acceleration vector of the end-effector $\ddot{X}$ and the acceleration vector of joint variables $\ddot{q}$. This formulation can be obtained by differentiating Equation (7) with respect to time:

$$
\ddot{q}=J^{-1} \ddot{X}+J_{q}^{-1}\left(\dot{J}_{X}-\dot{J}_{q} J^{-1}\right) \dot{X}
$$

\subsection{Dynamics}

The dynamics of the 4-DOF parallel robot can be computed using the Lagrangian approach together with some simplifications. The main hypothesis of the dynamic model is to consider the inertia of the small arm negligible and to approximate the distributed mass of each arm with two lumped masses positioned at the extremities of the link, as shown in Figure 4. A further hypothesis simplifies the two bars of the mobile platform, which is split into two parts that can only translate 
relative to each other, with two lumped masses located at each extremity, as reported in Figure 3. These lumped masses are indicated with $m_{C}=m_{D}=m_{\text {plat }} / 2+2 m_{\mathcal{C}}$ [37].

The inverse dynamics of the manipulator aims at calculating the torque required for the motion of the parallel robot by knowing the position, velocity and acceleration of the joint variables. The total torque $\tau$ required by the actuators is the sum of the contributes given by (a) the actuating system (actuators and upper arms) $\tau_{a c t}$, (b) the end-effector platform $\tau_{\text {plat }}$, and (c) the payload $\tau_{p}$. The first contribution can be written as:

$$
\boldsymbol{\tau}_{a c t}=\boldsymbol{I}_{a c t} \ddot{\boldsymbol{q}}+g \boldsymbol{M}_{\text {arm }} \cos (\boldsymbol{q})+f_{s}+\boldsymbol{F}_{v} \dot{\boldsymbol{q}}
$$

where $I_{a c t}=\operatorname{diag}\left(\left[\begin{array}{llll}I_{e q} & I_{e q} & I_{e q} & I_{e q}\end{array}\right]\right)$ is the inertia matrix, $I_{e q}=K_{r}^{2} I_{a c t}+I_{a}+I_{c}, K_{r}$ is the reduction ratio, $I_{a}=1 / 3 m_{a} r_{u, i}^{2}$ and $I_{c}=m_{c} r_{u, i}^{2}$. The matrix $\boldsymbol{M}_{\text {arm }}=\operatorname{diag}\left(\left[\begin{array}{llll}m_{e q} & m_{e q} & m_{e q} & m_{e q}\end{array}\right]\right)$, where the diagonal terms are expressed as $m_{e q}=m_{a} r_{u, i} / 2+m_{c} r_{u, i}$, comprises the contributions of the upper arm and the point mass. The constant $g$ represents the gravitational acceleration. Friction effects are taken into account with the Coulomb and viscous friction forces $f_{s}=f_{s} \operatorname{sign}(\dot{\boldsymbol{q}})$ and $\boldsymbol{F}_{\boldsymbol{v}}=$ $\operatorname{diag}\left(\left[\begin{array}{llll}f_{v} & f_{v} & f_{v} & f_{v}\end{array}\right]\right)$. Equation (11) can be rewritten by substituting $\ddot{q}$ with the expression of the inverse acceleration problem (Equation (10)) as function of the velocity $\dot{X}$ and acceleration $\ddot{X}$ of the end-effector as:

$$
\tau_{a c t}=\boldsymbol{I}_{a c t} \boldsymbol{J}^{-1} \ddot{\boldsymbol{X}}+\boldsymbol{I}_{a c t} \boldsymbol{J}_{v e l}{ }^{-1} \dot{\boldsymbol{X}}+g \boldsymbol{M}_{\text {arm }} \cos (\boldsymbol{q})+\boldsymbol{f}_{\boldsymbol{s}}+\boldsymbol{F}_{\boldsymbol{v}} \dot{\boldsymbol{q}}
$$

with $J_{v e l}{ }^{-1}=J_{q}{ }^{-1}\left(\dot{J}_{X}-\dot{J}_{q} J^{-1}\right)$.
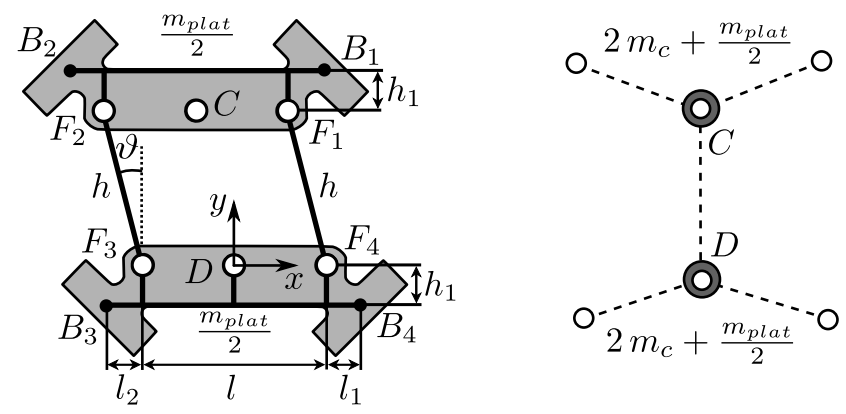

Figure 3. Model of the mobile platform.
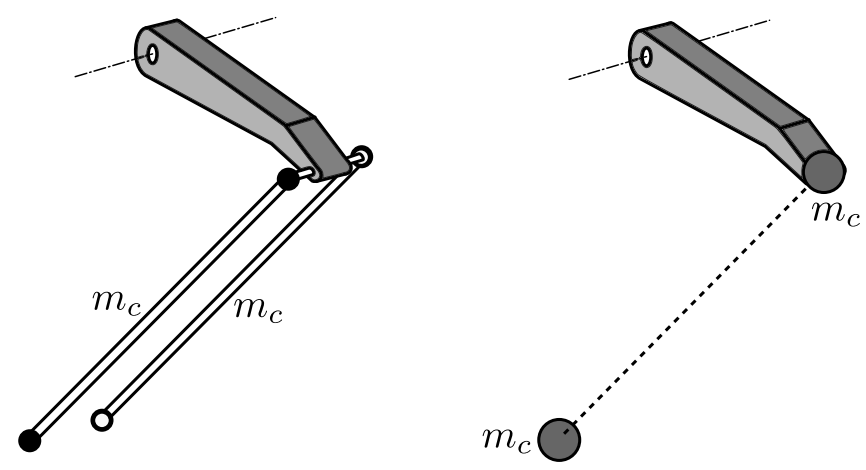

Figure 4. Model of the arm.

The second torque contribution, given by the mobile platform, can be expressed by:

$$
\tau_{\text {plat }}=\boldsymbol{J}^{T} \boldsymbol{M}_{\text {plat }} \ddot{\boldsymbol{X}}+2 \boldsymbol{J}^{T} \boldsymbol{M}_{\boldsymbol{C}} \boldsymbol{G}
$$

where matrices $\boldsymbol{M}_{\text {plat }}$ and $\boldsymbol{M}_{\boldsymbol{C}}$ account for the dynamic contributions of the point masses $\boldsymbol{C}$ and $\boldsymbol{D}$ (please see [16,37] for further details). 
The third torque contribution is given by the payload applied to the end-effector and can be written as:

$$
\boldsymbol{\tau}_{p}=\boldsymbol{J}^{T} \boldsymbol{M}_{\boldsymbol{p}}(\ddot{\mathbf{X}}+\boldsymbol{G})
$$

where matrix $\boldsymbol{M}_{p}$ contains on the main diagonal the mass $m_{p}$ and the inertia $I_{p}$ of the considered load.

At the end, the complete expression for the torque required by the robot actuators to steer the end-effector is given by:

$$
\boldsymbol{\tau}=\left[\boldsymbol{I}_{\text {act }} \boldsymbol{J}^{-1}+\boldsymbol{J}^{T}\left(\boldsymbol{M}_{\text {plat }}+\boldsymbol{M}_{\boldsymbol{p}}\right)\right] \ddot{\boldsymbol{X}}+\boldsymbol{J}^{T}\left(\boldsymbol{M}_{\text {plat }}+\boldsymbol{M}_{\boldsymbol{p}}\right) \boldsymbol{G}+\boldsymbol{I}_{\text {act }} \boldsymbol{J}_{\boldsymbol{v e l}}{ }^{-1} \dot{\boldsymbol{X}}+g \boldsymbol{M}_{\text {arm }} \cos (\boldsymbol{q})+\boldsymbol{f}_{\boldsymbol{s}}+\boldsymbol{F}_{\boldsymbol{v}} \dot{\boldsymbol{q}}
$$

Table 1. Geometrical parameters of the parallel robot.

\begin{tabular}{llll}
\hline Parameter & Value & Parameter & Value \\
\hline$O P_{i}$ & $0.275 \mathrm{~m}$ & $l$ & $0.081 \mathrm{~m}$ \\
$r_{u, i}$ & $0.375 \mathrm{~m}$ & $l_{1}$ & $0.044 \mathrm{~m}$ \\
$r_{l, i}$ & $0.825 \mathrm{~m}$ & $l_{2}$ & $0.025 \mathrm{~m}$ \\
$z_{P i}$ & $0.105 \mathrm{~m}$ & $h$ & $0.110 \mathrm{~m}$ \\
$z_{e}$ & $0.087 \mathrm{~m}$ & $h_{1}$ & $0.025 \mathrm{~m}$ \\
\hline
\end{tabular}

Table 2. Dynamic parameters of the parallel robot.

\begin{tabular}{llllll}
\hline Parameter & Symbol & Value & Parameter & Symbol & Value \\
\hline motor torque constant & $K_{t}$ & $0.99 \mathrm{Nm} / \mathrm{A}$ & reduction ratio & $K_{r}$ & 50 \\
motor back-emf constant & $K_{e}$ & $0.61 \mathrm{~V} \cdot \mathrm{s} / \mathrm{rad}$ & gearbox inertia & $I_{r}$ & $5.70 \cdot 10^{-6} \mathrm{Kg} \mathrm{m} \mathrm{m}^{2}$ \\
winding resistance & $R$ & $2.81 \Omega$ & upper arm mass & $m_{a}$ & $1.85 \mathrm{Kg}$ \\
motor inertia & $I_{m}$ & $1.62 \cdot 10^{-4} \mathrm{Kg} \mathrm{m}^{2}$ & point mass (arm) & $m_{c}$ & $0.24 \mathrm{Kg}$ \\
static friction coefficient & $f_{s}$ & $0.109 \mathrm{Nm}$ & platform mass & $m_{p l a t}$ & $3.50 \mathrm{Kg}$ \\
viscous friction coefficient & $f_{v}$ & $1.62 \cdot 10^{-4} \mathrm{Nm} \cdot \mathrm{s} / \mathrm{rad}$ & load mass & $m_{p}$ & $0.10 \mathrm{Kg}$ \\
driver efficiency & $\eta_{d}$ & 0.90 & load inertia & $I_{p}$ & $8.33 \cdot 10^{-4} \mathrm{Kg} \mathrm{m} \mathrm{m}^{2}$ \\
gravitational acceleration & $g$ & $9.81 \mathrm{~m} / \mathrm{s}^{2}$ & point mass (platform) & $m_{C}$ & $2.23 \mathrm{Kg}$ \\
\hline
\end{tabular}

\section{Electro-Mechanical Model}

The electro-mechanical model of the actuators of the robot is developed by assuming that brushless DC motors are used as in [23,31]. The torque exerted by the $j$-th motor $\tau_{m, j}=\tau_{j} / K_{r}$ is proportional to the current $i_{j}(t)$ drawn by the motor itself as:

$$
\tau_{m, j}(t)=K_{t} i_{j}(t)
$$

being $K_{t}$ the motor torque constant. Then, the armature model describes the voltage drop $v_{j}(t)$ across the $j$-th motor of the manipulator as follows:

$$
v_{j}(t)=R i_{j}(t)+K_{e} \dot{q}_{m, j}(t)
$$

where $R$ is the resistance of the motor windings, $K_{e}$ is the back-emf constant, whereas $\dot{q}_{m, j}=\dot{q}_{j} K_{r}$ indicates the velocity of the $j$-th motor shaft. By taking into account the efficiency of the driver $\eta_{d}$, the instantaneous electric power drawn by the $j$-th robot actuator is given by the voltage-current product:

$$
P_{e, j}(t)=\frac{v_{j}(t) i_{j}(t)}{\eta_{d}}
$$

The electric power $P_{e, j}$ takes positive values when the energy is drawn from the drive unit, whereas it takes negative values when the energy flows from the actuators to the drive unit. If regenerative drives are assumed, the instantaneous electric power can be divided into consumed power $P_{c, j}$ and braking recovered power $P_{r, j}\left(P_{e, j}=P_{c, j}-P_{r, j}\right)$, which are computed as follows:

$$
P_{c, j}=\left\{\begin{array}{ccc}
P_{e, j} & \text { if } \quad P_{e, j} \geq 0 \\
0 & \text { if } \quad P_{e, j}<0
\end{array} \quad P_{r, j}=\left\{\begin{array}{ccc}
0 & \text { if } \quad P_{e, j} \geq 0 \\
-P_{e, j} & \text { if } \quad P_{e, j}<0
\end{array}\right.\right.
$$


Finally, the overall energy consumption $E_{e}$, as well as the consumed and recovered energies $E_{c}$ and $E_{r}$, can be obtained by integrating the corresponding electric power of the four actuators of the robot over the time period $T$ :

$$
E_{e}=\sum_{j=1}^{4} \int_{0}^{T} P_{e, j} d t \quad E_{c}=\sum_{j=1}^{4} \int_{0}^{T} P_{c, j} d t \quad E_{r}=\sum_{j=1}^{4} \int_{0}^{T} P_{r, j} d t
$$

The regenerated energy can be stored in a capacitor or it can be shared by the other drivers connected to the same electrical bus. On the other hand, if the motor drives do not support regeneration [39], as it is assumed in this work, the current drawn from the actuators to the drive units during the deceleration phases is dissipated in a braking resistor. Therefore, only the contribution given by $P_{c}$ is considered.

\section{Task-Dependent Analysis}

In this section the task-dependent analysis of the 4-DOF parallel manipulator that is used in this paper as a method for the enhancement of the energetic performance of the robot is carried out. The analysis, which is based on the formulations of the dynamic and electro-mechanical models of the manipulators presented in Sections 2 and 3, takes into consideration a basic task: the translation of the end-effector along a linear path, defined by a fixed total displacement $L$. This task can be related to a common pick-and-place operation, which is commonly performed by high-speed parallel robots in industry. The rotation of the fourth degree of freedom $\vartheta$ is not taken into account in this work, since preliminary tests show a limited influence of $\vartheta$ on the overall energy consumption, due to the limited inertia of the load.

The motion law chosen for the execution of the assigned task is a trapezoidal speed profile, with a fixed total time $T=0.150 \mathrm{~s}$. The acceleration and deceleration times $t_{1}$ and $t_{3}$ are chosen equal to $T / 3$, a condition that corresponds to the minimum-energy solution for the chosen speed profile for constant inertia systems [21]. The trajectory is planned in the operational space in order to keep the length of the path fixed for the purposes of the energetic analysis.

Under these hypotheses, the path of the end-effector of the parallel manipulator can be located within the robot workspace in countless ways. Figure 5 shows a graphical overview of the parametrization chosen to represent the task in a plane $x O y$ parallel to the robot base. The center of the path is defined by the cylindrical coordinates $(d, \phi)$ of point $C$, where $d$ represents the distance between $C$ and the base of the robot $O$ and $\phi$ the angle between the $x$-axis and the segment $d$. Furthermore, the angle $\theta$ represents the orientation of the path with respect to the segment $d$. The end-effector coordinates in the operational space can be defined from the initial pose $P_{A}$ to the final pose $P_{B}$ as follows:

$$
\begin{aligned}
& P_{A}=\left[\begin{array}{llll}
c_{x}-r \cos (\theta+\phi) & c_{y}-r \sin (\theta+\phi) & z_{A} & \vartheta_{A}
\end{array}\right]^{T} \\
& P_{B}=\left[\begin{array}{llll}
c_{x}+r \cos (\theta+\phi) & c_{y}+r \sin (\theta+\phi) & z_{B} & \vartheta_{B}
\end{array}\right]^{T}
\end{aligned}
$$

where $C=\left[c_{x}, c_{y}\right]^{T}=[d \cos (\phi), d \sin (\phi)]^{T}, r=L / 2, z_{A}=z_{B}$, since the path is parallel to the robot base, and $\vartheta_{A}=\vartheta_{B}=0$.

The energy consumption maps, reported in the next section, are computed in three different cases, described in Table 3.

Table 3. Description of the three considered scenarios.

\begin{tabular}{cccccc}
\hline Case & $z[\mathrm{~m}]$ & $\boldsymbol{\phi}[\mathrm{rad}]$ & $\boldsymbol{\theta}[\mathrm{rad}]$ & $\boldsymbol{d}[\mathrm{m}]$ & $\boldsymbol{L}[\mathrm{m}]$ \\
\hline$(1)$ & -0.80 & {$[0, \pi / 2]$} & {$[0, \pi]$} & {$[0,0.650]$} & 0.150 \\
$(2)$ & {$[-0.730,-1.230]$} & 0.00 & {$[0, \pi]$} & {$[0,0.350]$} & 0.150 \\
$(3)$ & {$[-0.730,-1.230]$} & 0.00 & {$[0, \pi]$} & 0.00 & 0.350 \\
\hline
\end{tabular}




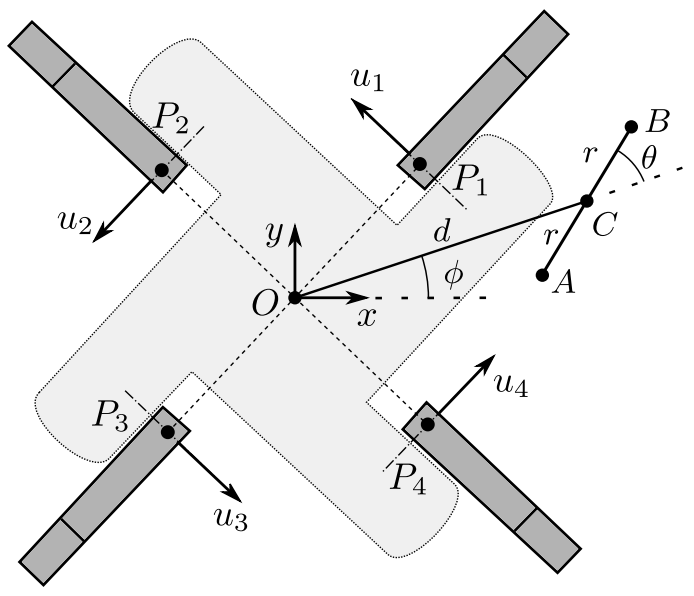

Figure 5. Top view of the path parametrization. Only the upper arms of the robot are shown.

Case (1) analyses the energy consumption of the manipulator in a plane with fixed distance from the robot base. This case applies when the task is bounded to be placed at a fixed distance $z$ from the robot base, e.g., when operating together with a conveyor belt. The variables of the problem are the angles $\phi$ and $\theta$, as well as the distance $d$. In particular, $d$ ranges from 0 to $0.650 \mathrm{~m}$, which represents the workspace limit in the upper part of the truncated cone (Figure 1). The angle $\phi$ spans over the range $[0, \pi / 2]$, whereas $\theta$ varies in $[0, \pi]$, to cover all possible solutions without repetitions imposed by the symmetry of the robot (Figure 5 ), even if the robot end-effector is not perfectly symmetrical with respect to the $\mathrm{x}$ and $\mathrm{y}$-axis. This introduces negligible differences in the consumed energy that, for the sake of simplicity, have not been considered in this work.

In Case (2) the angle $\phi$ is fixed and the analysis is carried out for several values of $z$, thus spanning operations performed at different heights. The angle $\theta$ and the distance $d$ are varied as well. In this case, $d$ is varied only between 0 and $0.350 \mathrm{~m}$, since this is the workspace bound in the lower part of the truncated cone. This scenario allows to evaluate the energetic performance of the parallel manipulator with respect to the vertical positioning of the task. In both Cases (1) and (2), the length of the path is equal to $L=0.150 \mathrm{~m}$.

Finally, Case (3) analyses the energetic expenditure in a simpler scenario, in which a path length equal to $L=0.350 \mathrm{~m}$ is considered. This path spans a half cylindrical volume that has a base equal to the area of the lower workspace of the robot. In this case, $\phi$ is fixed and the only variables taken into account are the vertical coordinate $z$ and the angle $\theta$. All tasks intersect the central point $(x, y)=(0,0)$, as $d$ is set to zero. The execution time is set to $T=0.150 \mathrm{~s}$ in all the three cases.

Figure 6 reports an example trajectory obtained for $\phi=0.00 \mathrm{rad}, d=0.325 \mathrm{~m}, \theta=\pi / 3 \mathrm{rad}$, and $z=-1.00 \mathrm{~m}$ fixed. The position, velocity, and acceleration of the $\mathrm{x}$ and $\mathrm{y}$ end-effector coordinates measured in the operative space are shown. Figure 7 reports the values of the kinematic variables of the actuated joints and the torque vectors when performing the very same trajectory.

\section{Results}

Numerical results are obtained by implementing the kinematic, dynamic, and electro-mechanical models of the parallel robot in Matlab, using a laptop running Windows 10 Pro with an Intel i7-8565U CPU and 16 GB of RAM. By considering the path parameterization described in Section 4, the task-related analysis allows to obtain the energy consumption maps reported in Figures 8-10. Each map takes about $20 \mathrm{~s}$ to be calculated by the software. Each point of the maps corresponds to the energy consumption of a path. Furthermore, in each map a red cross indicates the minimum-energy point.The energy consumption maps for Case (1) are reported in Figure 8, whereas Table 4 reports the minimum and maximum energy solutions for each of the maps. Figure 8 is split into six maps, each one corresponding to a different value of $\phi$ within the limited range $[0, \pi / 2]$. Spanning for the remaining 
range $[\pi, 2 \pi]$ would result in almost identical energy values, as a consequence of the symmetry of the task and of the robot design.

By looking at the maps of Figure 8 , it can be seen that for small and large values of the angle $\theta$, i.e., if the task is parallel to the segment $d$, the energy consumption increases by increasing the distance $d$. On the other hand, when the task is parallel to the $y$ axis, i.e., $\theta$ is close to $\pi / 2$, it is generally advised to operate far from the center of the operative space, as the overall energy consumption is smaller in such an area. The minimum-energy point is found for values of $\theta$ close to $1.7 \mathrm{rad}$ for all the cases, except for map (d), in which the minimum is located at $\theta$ close to $\pi$. The energy consumption for Case (1) within the six maps assumes a minimum in map (f) for $\phi_{\min }=1.57 \mathrm{rad}, \theta_{\min }=0.597 \mathrm{rad}$, and $d_{\min }=1.67 \mathrm{~m}$. The values of energy in this scenario assumes the value of $E_{\min }=8.01 \mathrm{~J}$. Table 4 also reports the maximum energy solutions and the percentage energy reduction obtained with the minimum-energy point with respect to the worst case scenario $E_{\max }$ for each map. The maximum percentage energy reduction is obtained in map (f) and it corresponds to a value of $68.7 \%$.

The energy consumption maps for Case (2) are shown in Figure 9. In these maps, the dependence of energy consumption on the vertical coordinate $z$ can be appreciated. In the first map, which represents a task located within the upper limit of the workspace, the minimum energy consumption $\left(E_{\min }=9.91 \mathrm{~J}\right)$ is achieved for the maximum value of $d$ and for $\theta=1.93$. By increasing the vertical distance between task and robot base, the energy consumption generally decreases, as shown in Table 5, and therefore it is advised to avoid working close to the upper limit of the workspace. When operating on planes far from the robot base, as in Figure $9 \mathrm{~d}-\mathrm{f}$, it is better to operate close to the axis of symmetry of the robot. The overall minimum-energy solution is found in map (f) for values of $z_{\min }=-1.23 \mathrm{~m}, d_{\min }=3.14 \mathrm{~m}$ and $\theta_{\min }=\pi \mathrm{rad}$ and takes the value $E_{\min }=6.12 \mathrm{~J}$, as reported in Table 5. The maximum percentage energy reduction with respect to the worst case of $E_{\max }$, equal to $20.2 \mathrm{~J}$, is obtained in the same map and reaches the value of $69.6 \%$.

The resulting map for Case (3) is shown in Figure 10, and the minimum and maximum energy solution are shown in Table 6 . This task refers to a $0.35 \mathrm{~m}$ translation centered in $(x, y)=(0,0)$; it spans a half cylinder by varying $z$ and $\theta$ in $[0, \pi]$. The data in Figure 10 show that the required energy generally decreases by increasing the vertical coordinate $z$ and that it takes lower values for low values of $\theta$. The minimum-energy point is found for $z_{\min }=-1.18 \mathrm{~m}, \theta_{\min }=0.00 \mathrm{rad}$ and corresponds to an energy expenditure of $E_{\min }=39.4 \mathrm{~J}$. It should be noted that the energy consumption map is not perfectly symmetrical with respect to $\theta=\pi / 2$, since the center point of the end-effector coordinates (point $D$ ) is not positioned in its center of mass, as shown in Figure 3. The percentage of energy reduction with respect to the worst case scenario $\left(E_{\max }=73.8 \mathrm{~J}\right)$ reaches the value of $46.6 \%$.

Table 4. Minimum and maximum energy solutions for Case (1). ${ }^{*}$ Energy reduction w.r.t. $E_{\max }$.

\begin{tabular}{|c|c|c|c|c|c|c|c|c|}
\hline map & $\phi[\mathrm{rad}]$ & $d_{\min }[m]$ & $\theta_{\min }[\mathrm{rad}]$ & $E_{\min }[J]$ & $d_{\max }[m]$ & $\theta_{\max }[\mathrm{rad}]$ & $E_{\max }[J]$ & En. red. $[\%]^{*}$ \\
\hline (a) & 0.000 & 0.650 & 1.73 & 8.11 & 0.650 & 0.000 & 22.8 & 64.4 \\
\hline (b) & 0.314 & 0.597 & 1.73 & 8.16 & 0.650 & 0.000 & 23.1 & 64.7 \\
\hline (c) & 0.628 & 0.358 & 1.60 & 9.00 & 0.650 & 0.000 & 23.8 & 62.2 \\
\hline (d) & 0.942 & 0.199 & 2.95 & 9.24 & 0.650 & 0.257 & 24.7 & 62.7 \\
\hline (e) & 1.26 & 0.610 & 1.73 & 8.56 & 0.650 & 0.192 & 25.1 & 65.9 \\
\hline (f) & 1.57 & 0.597 & 1.67 & 8.01 & 0.650 & 0.064 & 25.6 & 68.7 \\
\hline
\end{tabular}



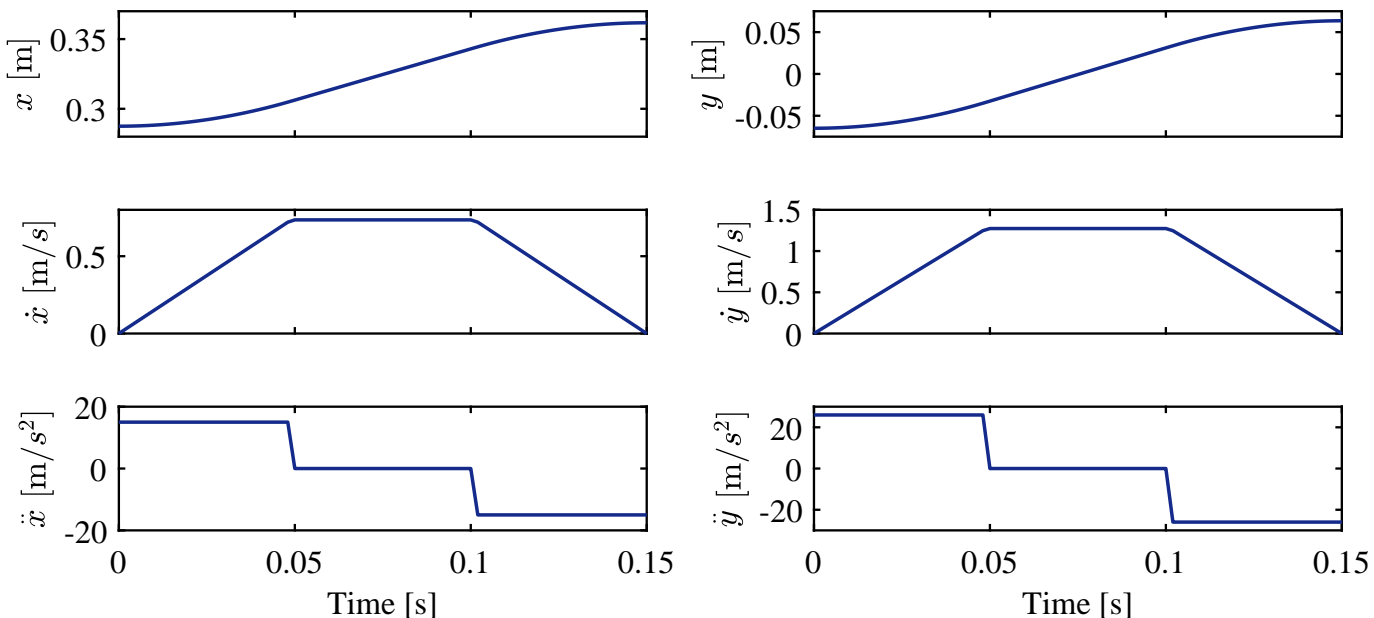

Figure 6. Example trajectory ( $\phi=0.00 \mathrm{rad}, d=0.325 m, \theta=\pi / 3 \mathrm{rad}$, and $z=-1.00 \mathrm{~m}$ fixed): position, velocity, and acceleration of the $\mathrm{x}$ and $\mathrm{y}$ end-effector coordinates ( $\vartheta$ are not taken into account).
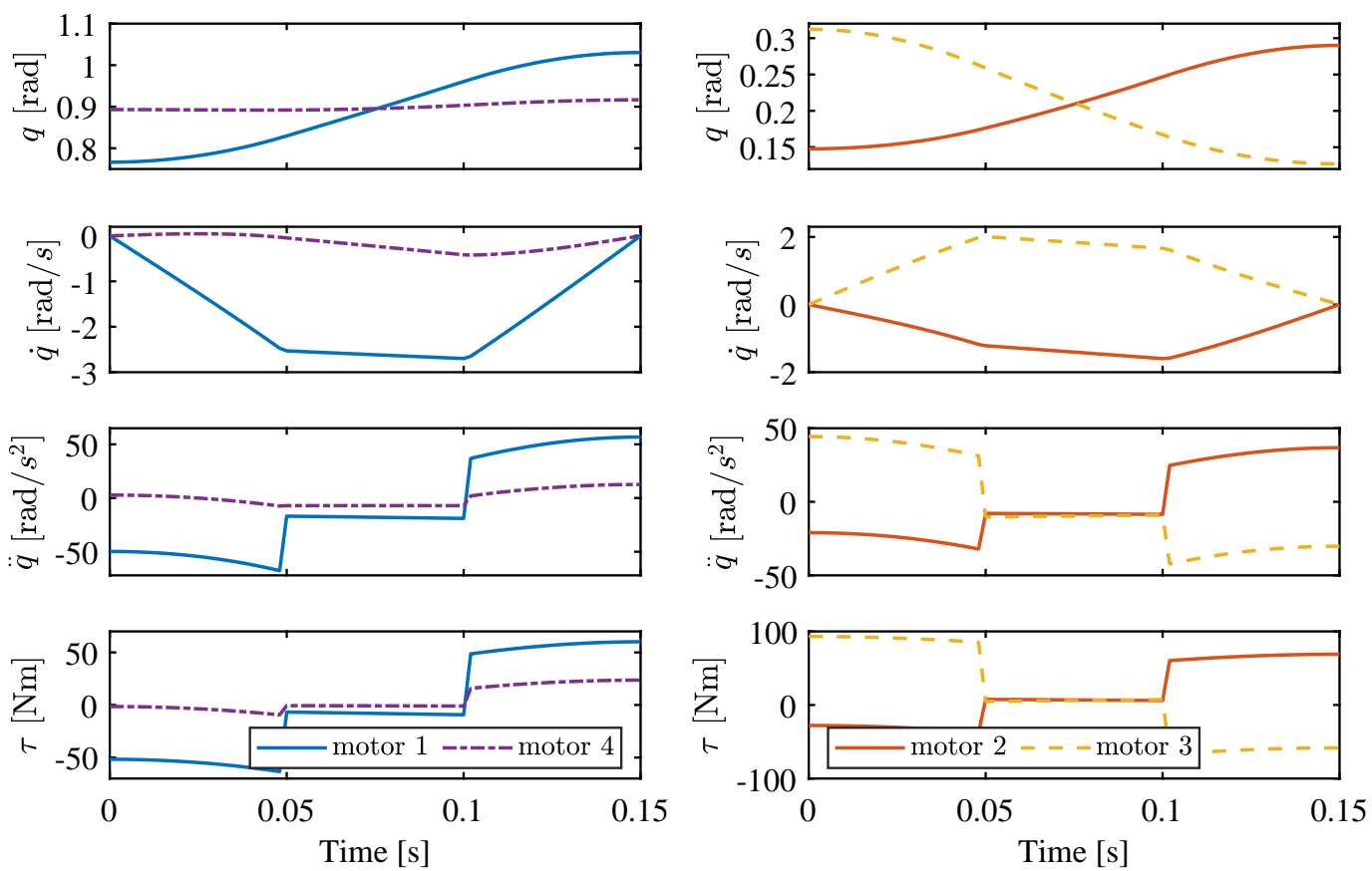

Figure 7. Example trajectory $(\phi=0.00 \mathrm{rad}, d=0.325 m, \theta=\pi / 3 \mathrm{rad}$, and $z=-1.00 \mathrm{~m})$ : position, velocity, acceleration, and torque of the actuated joints.

Table 5. Minimum and maximum energy solutions for Case (2). ${ }^{*}$ Energy reduction w.r.t. $E_{\max }$.

\begin{tabular}{|c|c|c|c|c|c|c|c|c|}
\hline Map & $z[m]$ & $d_{\min }[m]$ & $\theta_{\min }[\mathrm{rad}]$ & $E_{\min }[J]$ & $d_{\max }[m]$ & $\theta_{\max }[\mathrm{rad}]$ & $E_{\max }[J]$ & En. red. $[\%]^{*}$ \\
\hline (a) & -0.73 & 0.350 & 2.12 & 9.91 & 0.100 & 0.834 & 14.5 & 31.7 \\
\hline (b) & -0.83 & 0.350 & 1.92 & 8.43 & 0.350 & 0.000 & 11.0 & 23.1 \\
\hline (c) & -0.93 & 0.129 & 2.24 & 7.52 & 0.350 & 0.000 & 10.1 & 25.8 \\
\hline (d) & -1.03 & 0.000 & 0.77 & 6.70 & 0.350 & 0.000 & 10.1 & 33.4 \\
\hline (e) & -1.13 & 0.036 & 1.60 & 6.28 & 0.350 & 0.000 & 11.3 & 44.6 \\
\hline (f) & -1.23 & 0.086 & 3.14 & 6.12 & 0.350 & 0.000 & 20.2 & 69.6 \\
\hline
\end{tabular}




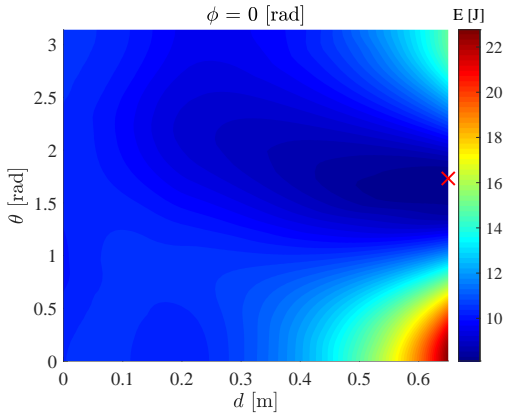

(a)

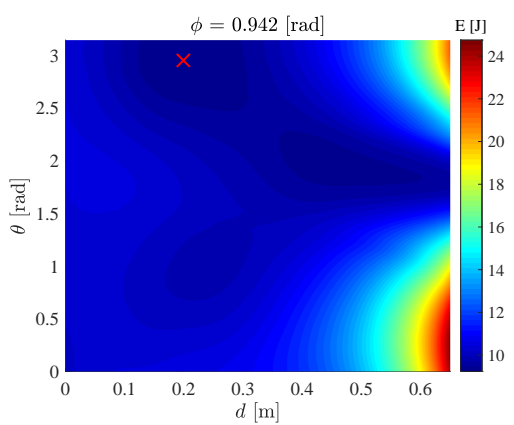

(d)

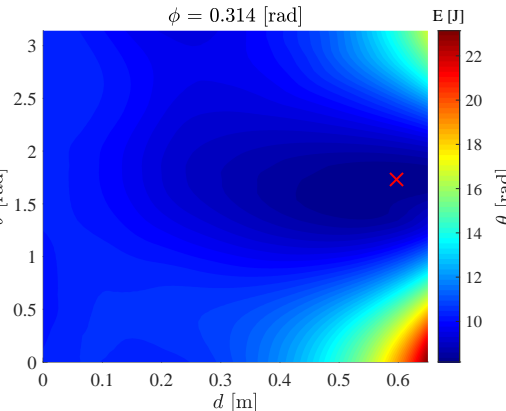

(b)

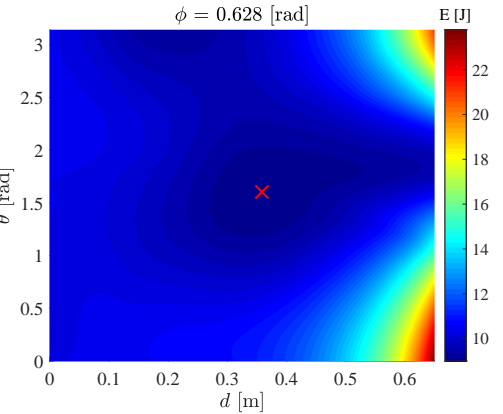

(c)

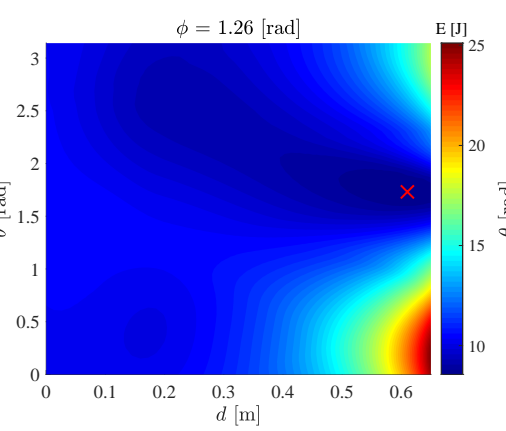

(e)

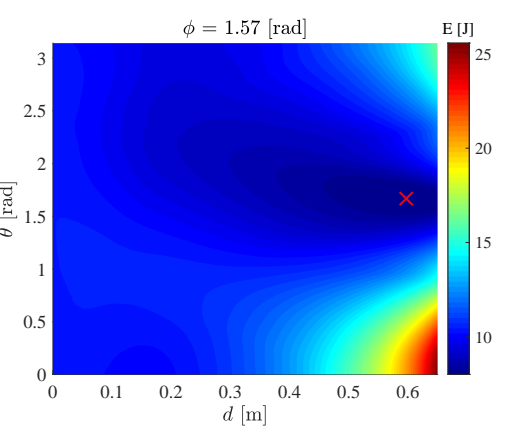

(f)

Figure 8. Energy consumption maps for Case (1).

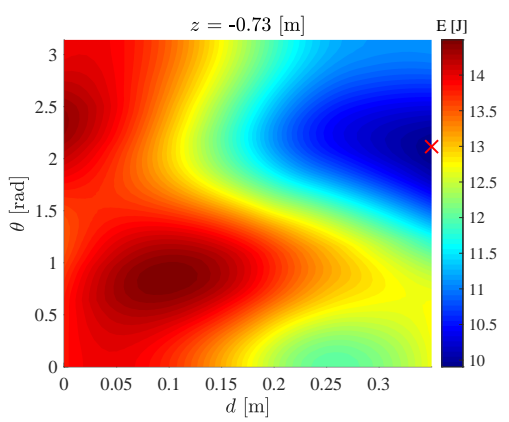

(a)

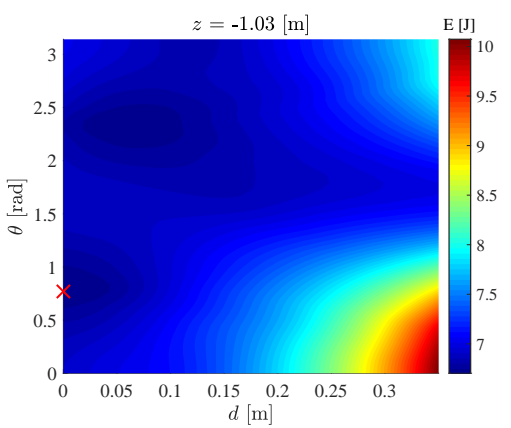

(d)

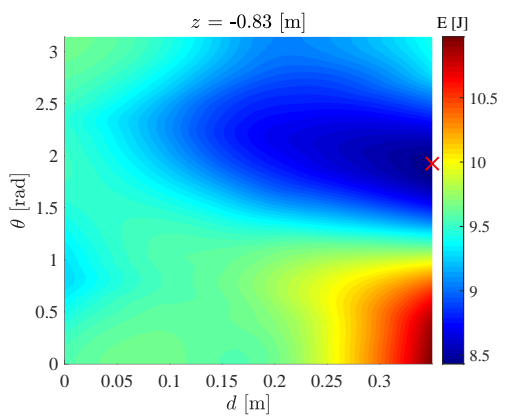

(b)

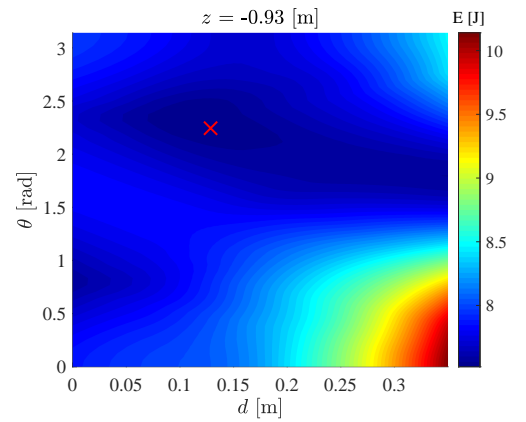

(c)

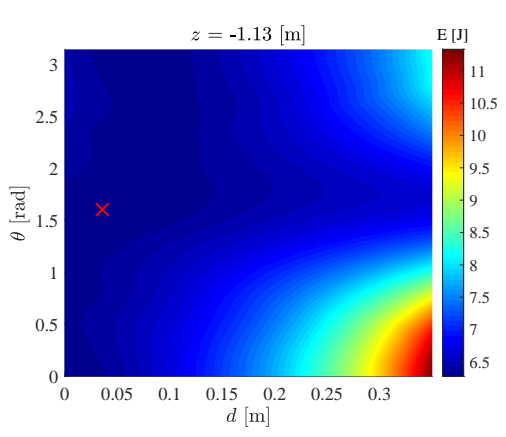

(e)

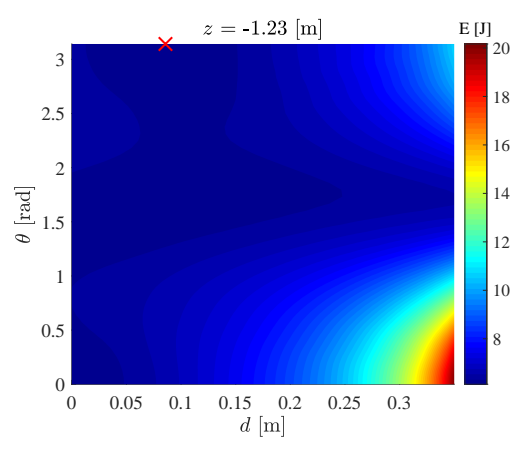

(f)

Figure 9. Energy consumption maps for Case (2).

Table 6. Minimum and maximum energy solution for Case (3). * Energy reduction w.r.t. $E_{\max }$.

\begin{tabular}{ccccccc}
\hline$z_{\min }[\mathrm{m}]$ & $\boldsymbol{\theta}_{\min }[\mathrm{rad}]$ & $\boldsymbol{E}_{\min }[\mathrm{J}]$ & $z_{\max }[\mathrm{m}]$ & $\boldsymbol{\theta}_{\max }[\mathrm{rad}]$ & $E_{\max }[\mathrm{J}]$ & En. red. [\% ${ }^{*}$ \\
\hline-1.18 & 0.000 & 39.4 & -0.730 & 2.05 & 73.8 & 46.6 \\
\hline
\end{tabular}




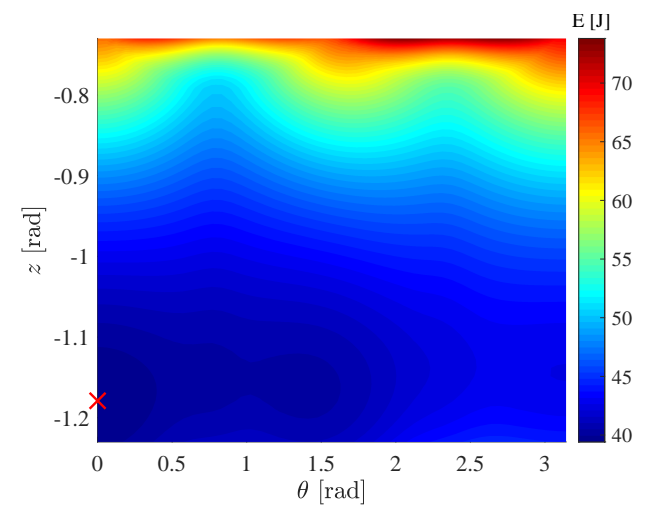

Figure 10. Energy consumption map for Case (3).

\section{Conclusions}

In this work a task-dependent energetic analysis of a 4-DOF parallel manipulator has been presented. The aim of the analysis is the estimation of the energetic performance of the robot when executing a simple task, i.e., a pick-and-place operation along a linear path. The task has been parametrized and the influence of the location of the starting and ending points within the robot workspace has been studied and summarized in energy consumption maps. The dynamic and electro-mechanical models of the manipulators have been developed and implemented to estimate the energy consumption of the robot actuators during the motion. Results indicate the best robot-task relative positioning, which can be applied in industrial environment for energy efficiency.

The numerical model implemented in this work can be used as an offline tool to guide the placement of a task within the robot workspace. The applicability of the method is general and the analysis can be extended to other tasks and to other manipulators as well.

Future developments of the work will see the implementation of an optimization routine to compute the minimum-energy location of a task within the whole robot workspace. Furthermore, future studies will consider the task-related analysis of more complex tasks and the extension of the study to the case of a task nonaligned with the base of the parallel manipulator.

Author Contributions: Conceptualization, L.S. and P.B.; methodology, software, validation, L.S., G.C. and P.B.; formal analysis, L.S., G.C. and P.B.; investigation, L.S. and P.B.; resources, R.V. and A.G.; data curation, L.S. and P.B.; writing-original draft preparation, L.S.; writing—review and editing, L.S. and P.B.; visualization, L.S. and P.B.; supervision, P.B., R.V. and A.G. All authors have read and agreed to the published version of the manuscript.

Funding: This research received no external funding.

Conflicts of Interest: The authors declare no conflict of interest.

\section{References}

1. European Comission. Proposal for a Directive of the European Parliament and of the Council Amending Directive 2012/27/EU on Energy Efficiency. Available online: https:/ / eur-lex.europa.eu/homepage.html (accessed on 14 January 2020).

2. Helm, D. The European framework for energy and climate policies. Energy Policy 2014, 64, 29-35. [CrossRef]

3. Kucukvar, M.; Cansev, B.; Egilmez, G.; Onat, N.C.; Samadi, H. Energy-climate-manufacturing nexus: New insights from the regional and global supply chains of manufacturing industries. Appl. Energy 2016, 184, 889-904. [CrossRef]

4. International Federation of Robotics. Executive Summary World Robotics 2019 Industrial Robots. Available online: https: / ifr.org/free-downloads / (accessed on 14 January 2020).

5. Carabin, G.; Wehrle, E.; Vidoni, R. A review on energy-saving optimization methods for robotic and automatic systems. Robotics 2017, 6, 39. [CrossRef] 
6. Li, Y.; Bone, G.M. Are Parallel Manipulators More Energy Efficient? In Proceedings of the 2001 IEEE International Symposium on Computational Intelligence in Robotics and Automation (Cat. No. 01EX515), Banff, AB, Canada, 29 July-1 August 2001; pp. 41-46.

7. Kim, Y.J. Design of Low Inertia Manipulator with HighStiffness and Strength using Tension Amplifying Mechanisms. In Proceedings of the IEEE/RSJ International Conference on Intelligent Robots and Systems (IROS), Hamburg, Germany, 28 September-2 October 2015; pp. 5850-5856.

8. Boscariol, P.; Gallina, P.; Gasparetto, A.; Giovagnoni, M.; Scalera, L.; Vidoni, R. Evolution of a Dynamic Model for Flexible Multibody Systems. In Advances in Italian Mechanism Science; Springer: Basel, Switzerland, 2017; pp. 533-541.

9. Yin, H.; Liu, J.; Yang, F. Hybrid structure design of lightweight robotic arms based on carbon fiber reinforced plastic and aluminum alloy. IEEE Access 2019, 7, 64932-64945. [CrossRef]

10. Vidoni, R.; Scalera, L.; Gasparetto, A. 3-D ERLS based dynamic formulation for flexible-link robots: theoretical and numerical comparison between the finite element method and the component mode synthesis approaches. Int. J. Mech. Control 2018, 19, 39-50.

11. Ghorbanpour, A.; Richter, H. Control With Optimal Energy Regeneration in Robot Manipulators Driven by Brushless DC Motors. In Proceedings of the ASME Dynamic Systems and Control Conference, Atlanta, GA, USA, 30 September-3 October 2018; p. V001T04A003.

12. Carabin, G.; Palomba, I.; Wehrle, E.; Vidoni, R. Energy Expenditure Minimization for a Delta-2 Robot Through a Mixed Approach. In Proceedings of the IFToMM World Congress on Mechanism and Machine Science, Krakow, Poland, 30 June-4 July 2019; pp. 383-390.

13. Khalaf, P.; Richter, H. Trajectory optimization of robots with regenerative drive systems: Numerical and experimental results. IEEE Trans. Robot. 2019. [CrossRef]

14. Scalera, L.; Palomba, I.; Wehrle, E.; Gasparetto, A.; Vidoni, R. Natural motion for energy saving in robotic and mechatronic systems. Appl. Sci. 2019, 9, 3516. [CrossRef]

15. Barreto, J.P.; Corves, B. Resonant Delta Robot for Pick-and-Place Operations. In Proceedings of the IFToMM World Congress on Mechanism and Machine Science, Krakow, Poland, 30 June-4 July 2019; pp. 2309-2318.

16. Scalera, L.; Carabin, G.; Vidoni, R.; Wongratanaphisan, T. Energy efficiency in a 4-DOF parallel robot featuring compliant elements. Int. J. Mech. Control 2019, 20, 49-57.

17. Paes, K.; Dewulf, W.; Vander Elst, K.; Kellens, K.; Slaets, P. Energy efficient trajectories for an industrial ABB robot. Proc. Cirp 2014, 15, 105-110. [CrossRef]

18. Ho, P.M.; Uchiyama, N.; Sano, S.; Honda, Y.; Kato, A.; Yonezawa, T. Simple motion trajectory generation for energy saving of industrial machines. SICE J. Control Meas. Syst. Integr. 2014, 7, 29-34. [CrossRef]

19. Boscariol, P.; Gasparetto, A.; Vidoni, R. Planning Continuous-Jerk Trajectories for Industrial Manipulators. In Proceedings of the ASME 2012 11th Biennial Conference on Engineering Systems Design and Analysis, Nantes, France, 2-4 July 2012; pp. 127-136.

20. Boscariol, P.; Carabin, G.; Gasparetto, A.; Lever, N.; Vidoni, R. Energy-efficient point-to-point trajectory generation for industrial robotic machines. In Proceedings of the ECCOMAS Thematic Conference on Multibody Dynamics, Barcelona, Spain, 29 June-2 July 2015; pp. 1425-1433.

21. Carabin, G.; Vidoni, R.; Wehrle, E. Energy Saving in Mechatronic Systems Through Optimal Point-to-Point Trajectory Generation Via Standard Primitives. In Proceedings of the International Conference of IFToMM ITALY, Cassino, Italy, 29-30 November 2018; pp. 20-28.

22. Ruiz, A.G.; Fontes, J.V.; da Silva, M.M. The Influence of Kinematic Redundancies in the Energy Efficiency of Planar Parallel Manipulators. In Proceedings of the ASME 2015 International Mechanical Engineering Congress and Exposition, Houston, TX, USA, 13-19 November 2015; p. V04AT04A010.

23. Boscariol, P.; Richiedei, D. Trajectory Design for Energy Savings in Redundant Robotic Cells. Robotics 2019, 8, 15. [CrossRef]

24. Shiller, Z. Time-Energy Optimal Control of Articulated Systems with Geometric Path Constraints. In Proceedings of the 1994 IEEE International Conference on Robotics and Automation, San Diego, CA, USA, 8-13 May 1994; pp. 2680-2685.

25. Trigatti, G.; Boscariol, P.; Scalera, L.; Pillan, D.; Gasparetto, A. A new path-constrained trajectory planning strategy for spray painting robots-rev.1. Int. J. Adv. Manuf. Technol. 2018, 98, 2287-2296. [CrossRef] 
26. Trigatti, G.; Boscariol, P.; Scalera, L.; Pillan, D.; Gasparetto, A. A Look-Ahead Trajectory Planning Algorithm for Spray Painting Robots with Non-Spherical Wrists. In Proceedings of the IFToMM Symposium on Mechanism Design for Robotics, Udine, Italy, 11-13 September 2018; pp. 235-242.

27. Kalawoun, R.; Lengagne, S.; Mezouar, Y. Optimal Robot Base Placements for Coverage Tasks. In Proceedings of the IEEE 14th International Conference on Automation Science and Engineering (CASE), Munich, Germany, 20-24 August 2018; pp. 235-240.

28. Scalera, L.; Seriani, S.; Gasparetto, A.; Gallina, P. Watercolour robotic painting: a novel automatic system for artistic rendering. J. Intell. Robot. Syst. 2019, 95, 871-886. [CrossRef]

29. Scalera, L.; Mazzon, E.; Gallina, P.; Gasparetto, A. Airbrush Robotic Painting System: Experimental Validation of a Colour Spray Model. In Proceedings of the International Conference on Robotics in Alpe-Adria Danube Region, Turin, Italy, 21-23 June 2017; pp. 549-556.

30. Boschetti, G. A picking strategy for circular conveyor tracking. J. Int. Robtic Syst. 2016, 81, $241-255$. [CrossRef]

31. Boscariol, P.; Scalera, L.; Gasparetto, A. Task-Dependent Energetic Analysis of a 3 dof Industrial Manipulator. In Proceedings of the International Conference on Robotics in Alpe-Adria Danube Region, Kaiserslautern, Germany, 19-21 June 2019; pp. 162-169.

32. Patel, S.; Sobh, T. Manipulator performance measures-a comprehensive literature survey. J. Int. Robtic Syst. 2015, 77, 547-570. [CrossRef]

33. Valsamos, C.; Wolniakowski, A.; Miatliuk, K.; Moulianitis, V.C. Optimal placement of a kinematic robotic task for the minimization of required joint velocities. Int. J. Mech. Control 2019, 20, 3-14.

34. Tanev, T.; Stoyanov, B. On the performance indexes for robot manipulators. Probl. Eng. Cybern. Robot. 2000, $49,64-71$.

35. Ur-Rehman, R.; Caro, S.; Chablat, D.; Wenger, P. Multi-objective path placement optimization of parallel kinematics machines based on energy consumption, shaking forces and maximum actuator torques: Application to the Orthoglide. Mech. Mach. Theory 2010, 45, 1125-1141. [CrossRef]

36. Boschetti, G.; Rosa, R.; Trevisani, A. Optimal robot positioning using task-dependent and direction-selective performance indexes: General definitions and application to a parallel robot. Robot. Comput.-Integr. Manuf. 2013, 29, 431-443. [CrossRef]

37. Pierrot, F.; Nabat, V.; Company, O.; Krut, S.; Poignet, P. Optimal design of a 4-DOF parallel manipulator: From academia to industry. IEEE Trans. Robot. 2009, 25, 213-224. [CrossRef]

38. Vidussi, F.; Boscariol, P.; Scalera, L.; Gasparetto, A. Energetic Analysis of Industrial Robots for Pick-and-Place Operations. In Proceedings of the 25th Jc-IFToMM Symposium (2019), 2nd Internationl Jc-IFToMM Symposium, Kanagawa, Japan, 26 October 2019.

39. Sayed-Ahmed, A.; Wei, L.; Seibel, B. Industrial Regenerative Motor-Drive Systems. In Proceedings of the 2012 Twenty-Seventh Annual IEEE Applied Power Electronics Conference and Exposition (APEC), Orlando, FL, USA, 5-9 February 2012; pp. 1555-1561.

(C) 2020 by the authors. Licensee MDPI, Basel, Switzerland. This article is an open access article distributed under the terms and conditions of the Creative Commons Attribution (CC BY) license (http://creativecommons.org/licenses/by/4.0/). 